\title{
Neutral atomic carbon in centers of galaxies
}

\author{
F. P. Israel ${ }^{1}$ and F. Baas ${ }^{1,2, \dagger}$ \\ 1 Sterrewacht Leiden, PO Box 9513, 2300 RA Leiden, The Netherlands \\ 2 Joint Astronomy Centre, 660 N. A'ohoku Pl., Hilo, Hawaii, 96720, USA
}

Received 27 June 2001 / Accepted 30 November 2001

\begin{abstract}
We present measurements of the emission from the centers of fifteen spiral galaxies in the ${ }^{3} \mathrm{P}_{1}-{ }^{3} \mathrm{P}_{0}[\mathrm{CI}]$ fine-structure transition at $492 \mathrm{GHz}$. Observed galaxy centers range from quiescent to starburst to active. The intensities of neutral carbon, the $J=2-1$ transition of ${ }^{13} \mathrm{CO}$ and the $J=4-3$ transition of ${ }^{12} \mathrm{CO}$ are compared in matched beams. Most galaxy centers emit more strongly in [CI] than in ${ }^{13} \mathrm{CO}$, completely unlike the situation pertaining to Galactic molecular cloud regions. [CI] intensities are lower than, but nevertheless comparable to $J=4-3{ }^{12} \mathrm{CO}$ intensities, again rather different from Galactic sources. The ratio of $[\mathrm{CI}]$ to ${ }^{13} \mathrm{CO}$ increases with the central [CI] luminosity of a galaxy; it is lowest for quiescent and mild starburst centers, and highest for strong starburst centers and active nuclei. Comparison with radiative transfer model calculations shows that most observed galaxy centers have neutral carbon abundances close to, or exceeding, carbon monoxide abundances, rather independent from the assumed model gas parameters. The same models suggest that the emission from neutral carbon and carbon monoxide, if assumed to originate in the same volumes, arises from a warm and dense gas rather than a hot and tenuous, or a cold and very dense gas. The observed [CI] intensities together with literature [CII] line and far-infrared continuum data likewise suggest that a significant fraction of the emission originates in medium-density gas $\left(n=10^{3}-10^{4} \mathrm{~cm}^{-3}\right)$, subjected to radiation fields of various strengths.
\end{abstract}

Key words. galaxies: ISM - ISM: molecules - radio lines: galaxies

\section{Introduction}

Carbon monoxide (CO), the most common molecule after $\mathrm{H}_{2}$, is now routinely detected in external galaxies. However, when exposed to energetic radiation, CO is readily photodissociated turning atomic carbon into an important constituent of the interstellar medium. As the ionization potential of neutral carbon is quite close to the dissociation energy of CO, neutral carbon subsequently may be ionized rather easily. As a consequence, [CI] emission primarily arises from interface regions between zones emitting in [CII] and CO respectively (see e.g. Israel et al. 1996; Bolatto et al. 2000). It requires column densities sufficiently high for shielding against ionizing radiation, but not so high that $\mathrm{CO}$ selfshielding allows most gas-phase carbon to be bound in molecules. In principle, observations of emission from $\mathrm{CO}, \mathrm{C}^{\circ}$ and $\mathrm{C}^{+}$provide significant information on the physical condition of the cloud complexes from which the emission arises (Israel et al. 1998; Gerin \& Phillips 2000; Israel \& Baas 2001). Even

Send offprint requests to: F. P. Israel, e-mail: israel@strw.leidenuniv.nl $\dagger$ Deceased April 4, 2001. though the far-infrared continuum and the [CII] line are much more efficient coolants, the various $\mathrm{CO}$ and [CI] lines are important coolants for relatively cool, dense molecular gas, contributing about equally to its cooling (Israel et al. 1995; Gerin \& Phillips 2000). In galaxies, however, studies of the dense interstellar medium are complicated by the effectively very large linear observing beams which incorporate whole ensembles of individual, mutually different clouds. The clumpy nature of the interstellar medium allows UV radiation to penetrate deeply into cloud complexes, so that the $\mathrm{CO},[\mathrm{CI}]$ and $[\mathrm{CII}]$ emitting volumes appear to coincide when observed with large beamsizes. The physics and structure of such photon-dominated regions (PDR's) has been reviewed most recently by Hollenbach \& Tielens (1999), whereas their consequent observational parameters have been modelled by e.g. Kaufman et al. (1999).

[CII] emission has been observed towards numerous galaxies, both from airborne (the now defunct NASA Kuiper Airborne Observatory) and from spaceborne (the equally defunct Infrared Space Observatory) platforms. In contrast to these [CII] observations, observations of [CI] emission can be performed on the ground, at least 
NGC 253

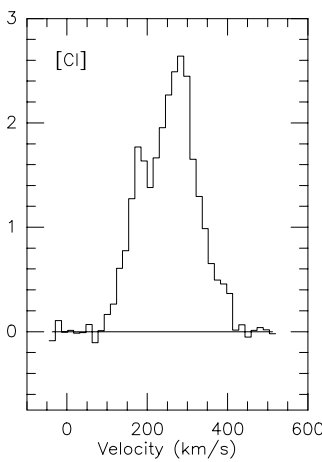

NGC 278

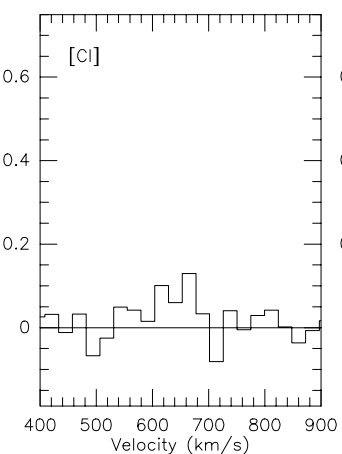

Maffei 2

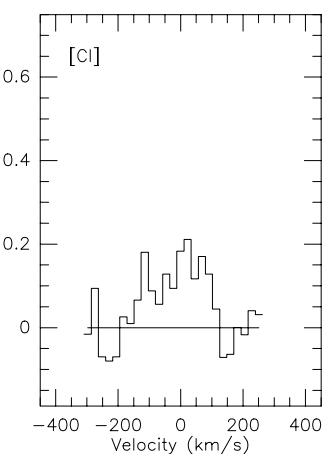

IC 342
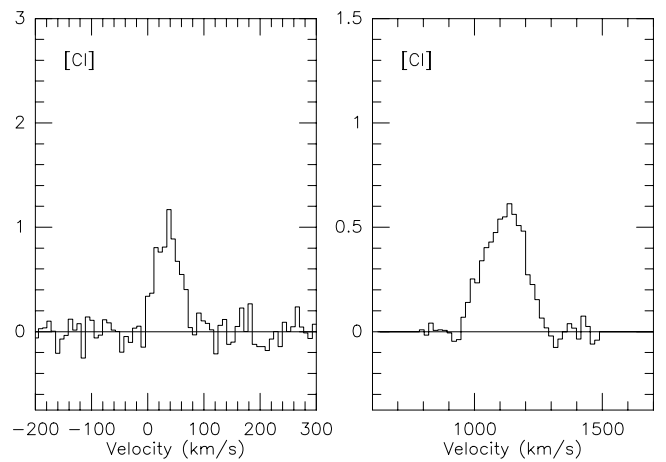
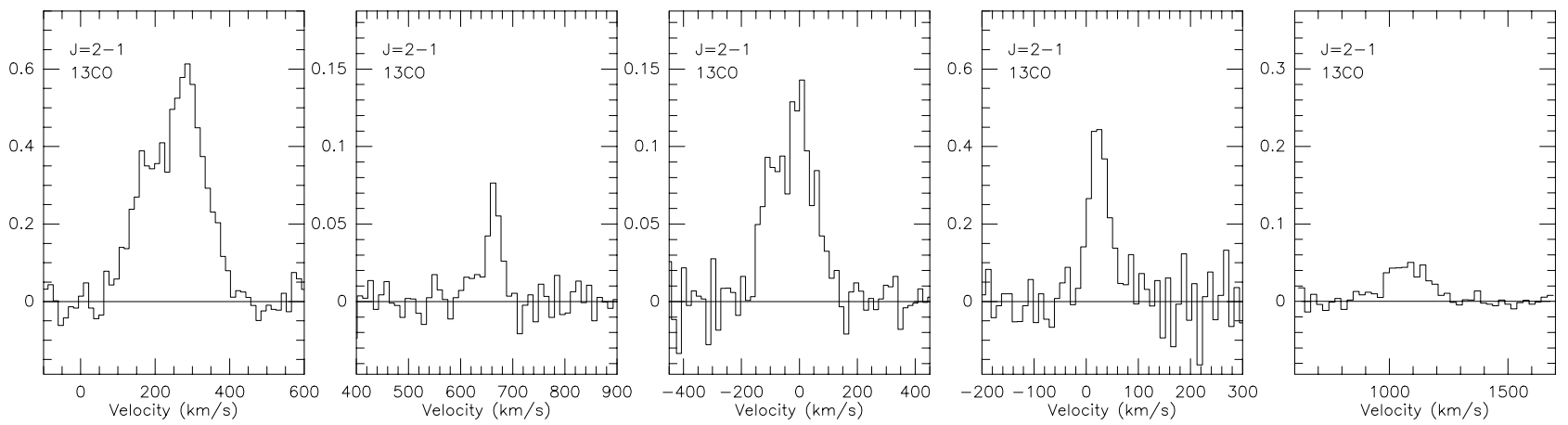

Fig. 1. [CI] and $J=2-1{ }^{13} \mathrm{CO}$ spectra observed towards sample galaxies. The vertical scale is $T_{\mathrm{mb}}$ in $\mathrm{K}$; the horizontal scale is velocity $V_{\mathrm{LSR}}$ in $\mathrm{km} \mathrm{s}^{-1}$. For all galaxies, the temperature range in $[\mathrm{CI}]$ is four times that in ${ }^{13} \mathrm{CO}$.

Table 1. Line observations log.

\begin{tabular}{|c|c|c|c|c|c|c|c|c|c|c|}
\hline \multirow[t]{2}{*}{ Galaxy } & \multicolumn{2}{|c|}{ Position } & \multirow{2}{*}{$\begin{array}{c}\text { Adopted } \\
\text { Distance } \\
(\mathrm{Mpc})\end{array}$} & \multicolumn{3}{|c|}{$[\mathrm{CI}]$} & \multicolumn{2}{|c|}{$J=2-1^{13} \mathrm{CO}$} & \multicolumn{2}{|c|}{$J=4-3{ }^{12} \mathrm{CO}$} \\
\hline & $\begin{array}{c}\mathrm{RA}(1950) \\
(\mathrm{h} \mathrm{m} \mathrm{s})\end{array}$ & $\begin{array}{c}\operatorname{Dec}(1950) \\
\left({ }^{\circ} \prime \prime \prime \prime\right)\end{array}$ & & $\begin{array}{r}\text { No. [CI] } \\
\text { Points }\end{array}$ & Date & $\begin{array}{l}T_{\text {sys }} \\
(\mathrm{K})\end{array}$ & Date & $\begin{array}{l}T_{\mathrm{sys}} \\
(\mathrm{K})\end{array}$ & Date & $\begin{array}{l}T_{\text {sys }} \\
(\mathrm{K})\end{array}$ \\
\hline NGC 253 & $00: 45: 05.7$ & $-25: 33: 38$ & 2.5 & 20 & $12 / 93$ & 3770 & $12 / 93$ & 1695 & $11 / 94$ & 9800 \\
\hline NGC 278 & $00: 49: 15.0$ & $+47: 16: 46$ & 12 & 1 & $07 / 96$ & 3650 & $06 / 95$ & 480 & $01 / 01$ & 1325 \\
\hline NGC 660 & $01: 40: 21.6$ & $+13: 23: 41$ & 13 & 1 & $07 / 96$ & 3065 & $05 / 01$ & 350 & $08 / 99$ & 3870 \\
\hline Maffei 2 & 02:38:08.5 & $+59: 23: 24$ & 2.7 & 1 & $12 / 93$ & 4885 & $01 / 96$ & 550 & $07 / 96$ & 3700 \\
\hline NGC 1068 & 02:40:07.2 & $-00: 13: 30$ & 14.4 & 22 & $07 / 96$ & 4000 & $01 / 96$ & 455 & $07 / 96$ & 3365 \\
\hline IC 342 & $03: 41: 36.6$ & $+67: 56: 25$ & 1.8 & 27 & $11 / 94$ & 4485 & $02 / 89$ & 1440 & $04 / 94$ & 2170 \\
\hline M 82 & 09:51:43.9 & $+69: 55: 01$ & 3.25 & 6 & $12 / 93$ & 7200 & $04 / 93$ & 335 & $10 / 93$ & 9085 \\
\hline NGC 3079 & 09:58:35.4 & $+55: 55: 11$ & 18.0 & 7 & $03 / 94$ & 6240 & $06 / 95$ & 310 & $03 / 94$ & 5510 \\
\hline NGC 3628 & $11: 17: 41.6$ & $+13: 51: 40$ & 6.7 & 8 & $11 / 94$ & 3450 & $06 / 95$ & 325 & $03 / 94$ & 2414 \\
\hline NGC 4826 & $12: 54: 17.4$ & $+21: 57: 06$ & 5.1 & 2 & $03 / 97$ & 3520 & $12 / 93$ & 535 & $12 / 93$ & 2045 \\
\hline M 51 & $13: 27: 45.3$ & $+47: 27: 25$ & 9.6 & 4 & $11 / 94$ & 6600 & $06 / 95$ & 370 & $04 / 96$ & 4065 \\
\hline M 83 & $13: 34: 11.3$ & $-29: 36: 39$ & 3.5 & 14 & $12 / 93$ & 4590 & $06 / 95$ & 430 & $12 / 93$ & 4360 \\
\hline NGC 5713 & $14: 37: 37.6$ & $-00: 04: 34$ & 21.0 & 1 & $02 / 99$ & 8000 & $12 / 00$ & 515 & $\ldots$ & $\ldots$ \\
\hline NGC 6946 & $20: 33: 48.8$ & $+59: 58: 50$ & 5.5 & 17 & $07 / 96$ & 3970 & $01 / 96$ & 530 & $07 / 96$ & 2900 \\
\hline NGC 7331 & $22: 34: 46.6$ & $+34: 09: 21$ & 14.3 & 1 & $11 / 96$ & 1925 & $12 / 97$ & 320 & $\ldots$ & $\ldots$ \\
\hline
\end{tabular}

in the ${ }^{3} \mathrm{P}_{1}-{ }^{3} \mathrm{P}_{0}[\mathrm{CI}]$ transition at $492 \mathrm{GHz}$. However, atmospheric transparency is poor at such high frequencies and weather conditions need to be unusually favourable for observations of the often weak extragalactic [CI] emission to succeed, even at the excellent high-altitude site of telescopes as the JCMT and the CSO. Consequently, the number of published results is relatively limited. Beyond the Local Group, i.e. at distances larger than $1 \mathrm{Mpc}$, [CI] has been mapped in bright galaxies such as IC 342 (Büttgenbach et al. 1992), M 82 (Schilke et al. 1993; White et al. 1994; Stutzki et al. 1997) and NGC 253 (Israel et al. 1995; Harrison et al. 1995), as well as M 83 (Petitpas \& Wilson 1998; Israel \& Baas 2001) and NGC 6946 (Israel \& Baas 2001). A survey of 13 galaxies, including limited 

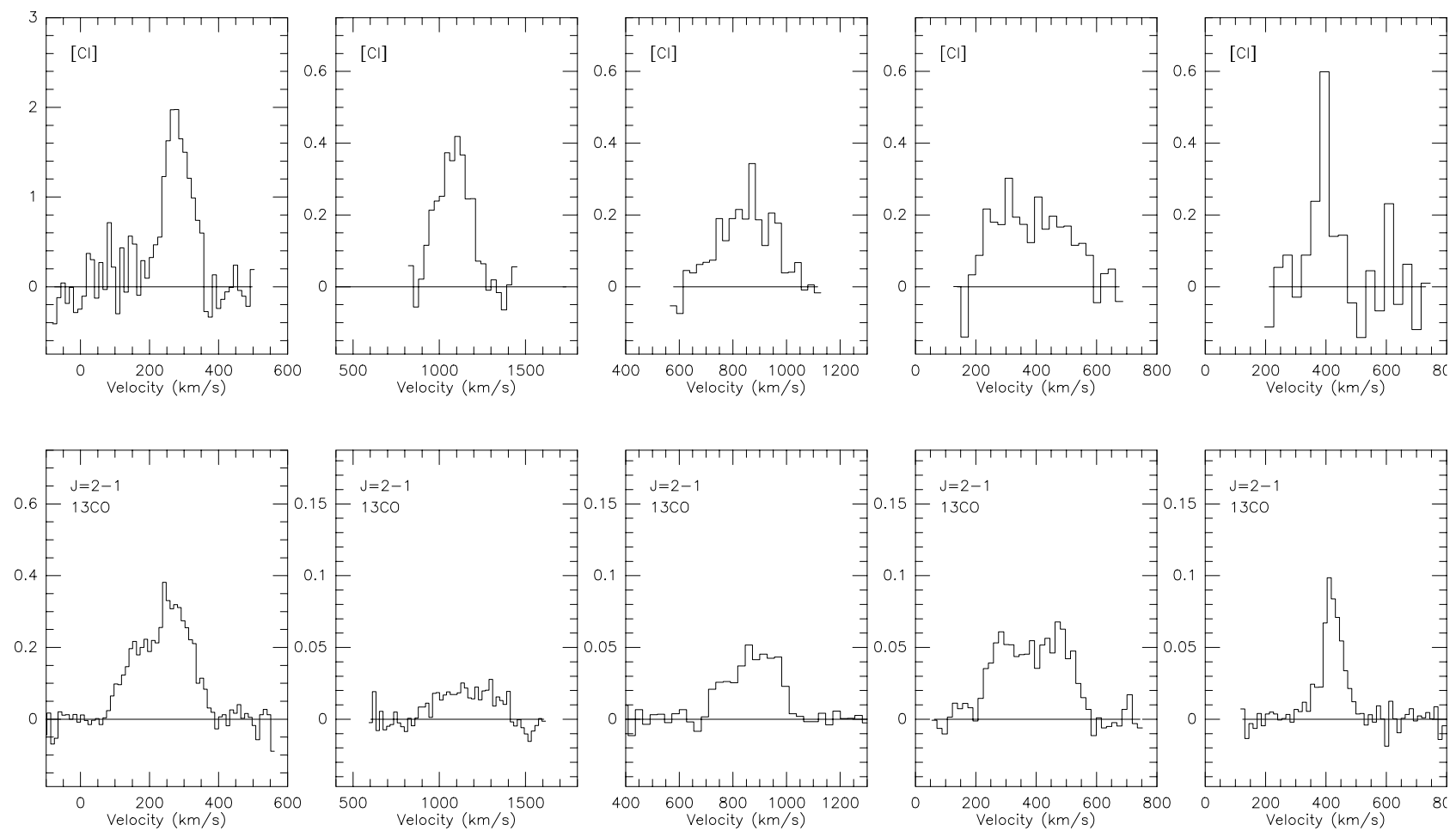

Fig. 1. continued.

Table 2. [CI], $J=2-1{ }^{13} \mathrm{CO}, J=4-3{ }^{12} \mathrm{CO}$ intensities.

\begin{tabular}{|c|c|c|c|c|c|c|c|}
\hline \multirow{2}{*}{$\begin{array}{l}\text { Galaxy } \\
\qquad \text { NGC } 253\end{array}$} & \multirow{2}{*}{$\begin{array}{c}\text { Offset } \\
\quad " \\
0,0\end{array}$} & \multirow{2}{*}{$\begin{array}{c}T_{\mathrm{mb}}([\mathrm{CI}]) \\
\left(10^{\prime \prime}\right) \\
(\mathrm{mK}) \\
2615\end{array}$} & \multicolumn{3}{|c|}{ 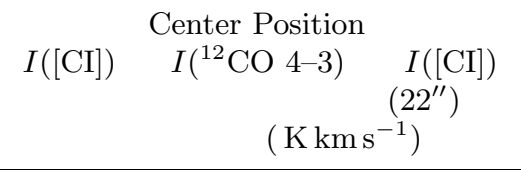 } & $I\left({ }^{13} \mathrm{CO}\right)$ & \multirow{2}{*}{$\begin{array}{l}\text { Area-integrated } \\
\text { [CI] Luminosity } \\
\left(\mathrm{K} \mathrm{km} \mathrm{s}^{-1} \mathrm{kpc}^{2}\right) \\
14 \pm 2.2\end{array}$} \\
\hline & & & $486 \pm 60$ & $1019 \pm 120$ & $290 \pm 45$ & $106 \pm 13$ & \\
\hline NGC 278 & 0,0 & 100 & $7 \pm 3$ & $9 \pm 2$ & $(5 \pm 1)$ & $2.6 \pm 0.4$ & $(3.1 \pm 0.7)$ \\
\hline NGC 660 & 0,0 & 240 & $50 \pm 8$ & $85 \pm 12$ & $(31 \pm 8)$ & $7.8 \pm 1.1$ & $(26 \pm 7)$ \\
\hline Maffei 2 & 0,0 & 190 & $37 \pm 7$ & $405 \pm 50$ & $(20 \pm 7)$ & $22 \pm 4$ & $(0.9 \pm 0.3)$ \\
\hline NGC 1068 & 0,0 & 560 & $109 \pm 12$ & $153 \pm 19$ & $49 \pm 9$ & $11 \pm 2$ & $53 \pm 8.5$ \\
\hline IC 342 & 0,0 & 1030 & $54 \pm 6$ & $209 \pm 21$ & $27 \pm 7$ & $24 \pm 3$ & $1.1 \pm 0.3$ \\
\hline M 82 & 0,0 & 2130 & $224 \pm 35$ & $591 \pm 95$ & $180 \pm 30$ & $60 \pm 9$ & $39 \pm 6.9$ \\
\hline NGC 3079 & 0,0 & 530 & $111 \pm 18$ & $115 \pm 20$ & $(70 \pm 15)$ & $12 \pm 3$ & $143 \pm 31$ \\
\hline NGC 3628 & $-17,+5$ & 265 & $84 \pm 11$ & $110 \pm 15$ & $38 \pm 8$ & $10 \pm 2$ & $28 \pm 5.7$ \\
\hline \multirow[t]{2}{*}{ NGC 4826} & 0,0 & 135 & $11 \pm 2$ & $\ldots$ & $17 \pm 4$ & $7.8 \pm 1.6$ & $\ldots$ \\
\hline & $-20,+5$ & 270 & $86 \pm 10$ & $73 \pm 9$ & $(47 \pm 10)$ & $15 \pm 2$ & $(13 \pm 3)$ \\
\hline \multirow[t]{4}{*}{ M 51} & 0,0 & 565 & $28 \pm 5$ & $24 \pm 4$ & $(13 \pm 4)$ & $8.4 \pm 1.9$ & $(8 \pm 2.7)$ \\
\hline & $-12,-12$ & 340 & $24 \pm 5$ & $\ldots$ & ... & $\ldots$ & $\ldots$ \\
\hline & $-12,-24$ & 755 & $55 \pm 9$ & $\ldots$ & $\ldots$ & $\ldots$ & $\ldots$ \\
\hline & $-24,-24$ & 470 & $55 \pm 9$ & $\ldots$ & $\ldots$ & $\ldots$ & $\ldots$ \\
\hline M 83 & 0,0 & 685 & $83 \pm 14$ & $270 \pm 20$ & $55 \pm 8$ & $29 \pm 3$ & $3.6 \pm 0.5$ \\
\hline NGC 5713 & 0,0 & $<90$ & $2 \pm 0.4$ & $\ldots$ & $\ldots$ & $7.4 \pm 1.6$ & $1.7-2.6$ \\
\hline NGC 6946 & 0,0 & 465 & $85 \pm 9$ & $216 \pm 20$ & $44 \pm 8$ & $22 \pm 3$ & $13 \pm 2.4$ \\
\hline NGC 7331 & 0,0 & 30 & $2 \pm 0.3$ & $\ldots$ & $\ldots$ & $2.5 \pm 0.6$ & $0.8-1.7$ \\
\hline
\end{tabular}

Note: Offset positions $(-17,+5)$ of NGC 3628 and $(-20,+5)$ of NGC 4826 are actual nucleus positions. 
radial mapping of NGC 891 and NGC 6946 was recently published by Gerin \& Phillips (2000). In this paper, we present a similar [CI] survey of 15 galaxies. We also obtained $J=2-1{ }^{13} \mathrm{CO}$ measurements for all galaxies, and $J=4-3$ measurements for all but two. Taking overlap into account, this survey brings the total number of galaxies outside the Local Group, detected in [CI], to 26 .

\section{Observations}

All observations were carried out with the $15 \mathrm{~m}$ James Clerk Maxwell Telescope (JCMT) on Mauna Kea

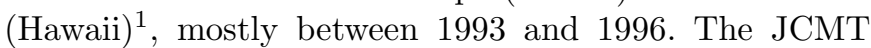
has a pointing accuracy better than $2^{\prime \prime}$ rms Observations of the ${ }^{3} \mathrm{P}_{1}-{ }^{3} \mathrm{P}_{0}[\mathrm{CI}]$ transition at $\nu=492.161 \mathrm{GHz}$ were made at a resolution of $10.2^{\prime \prime}$ (HPBW); those of the $J=$ $2-1{ }^{13} \mathrm{CO}$ transition with a resolution of $22^{\prime \prime}$. The observing conditions can be judged from the total system temperatures (including sky) which are listed in Table 1.

All observations were obtained using the Digital Autocorrelator Spectrograph as a backend. Spectra were binned to various resolutions; we applied linear baseline corrections only and scaled the spectra to main-beam brightness temperatures, $T_{\mathrm{mb}}=T_{\mathrm{A}}^{*} / \eta_{\mathrm{mb}}$. Line parameters were determined by Gaussian fitting and by adding channel intensities over the relevant range. For observations at $492 \mathrm{GHz}$ we used $\eta_{\mathrm{mb}}=0.43$ up to January 1995 and $\eta_{\mathrm{mb}}=0.50$ for later observations. For observations at $220 \mathrm{GHz}$, we used $\eta_{\mathrm{mb}}=0.69$. A list of the observed galaxies and additional information is given in Table 1.

\section{Results}

Observational results are summarized in Table 2; a number of representative profiles of both [CI] and $J=2-$ $1{ }^{13} \mathrm{CO}$ emission is shown in Fig. 1. For half of the galaxy sample, the distribution of [CI] was mapped beyond the central position (cf. Table 1). The [CI] emission maps of NGC 6946 and M 83 have already been published (Israel \& Baas 2001); the remaining maps will be discussed in forthcoming papers. In the meantime, we have used the information contained in the maps to convolve the fullresolution central [CI] intensities (Col. 4 of Table 2) to [CI] intensities (Col. 5) appropriate to the twice larger beamsize of the $J=2-1{ }^{13} \mathrm{CO}$ observations. We have likewise used the map information to determine total [CI] luminosities (Col. 7) integrated over the entire central source extent.

For all but two of the sample galaxies we have also maps of the $J=4-3{ }^{12} \mathrm{CO}$ distribution. Again, we refer to published and forthcoming papers for a discussion of these observations. For those galaxies that were not mapped in

1 The James Clerk Maxwell Telescope is operated by The Joint Astronomy Centre on behalf of the Particle Physics and Astronomy Research Council of the UK, The Netherlands Organisation for Scientific Research, and the National Research Council of Canada.
[CI], we have used the $J=4-3{ }^{12} \mathrm{CO}$ maps to estimate the convolved [CI] intensity and the total luminosity by assuming identical [CI] and $J=4-3{ }^{12} \mathrm{CO}$ distributions. This was shown to be the case for M 83 and NGC 6946 (Petitpas \& Wilson 1998; Israel \& Baas 2001), but we have further verified the validity of this assumption for all galaxies that were mapped in both [CI] and $J=4-3{ }^{12} \mathrm{CO}$. Values obtained in this way are given in parentheses in Table 2.

In all galaxies mapped, the central neutral carbon peak is well contained within a radius $R \leq 0.6 \mathrm{kpc}$, often as small as $R \approx 0.3 \mathrm{kpc}$. For only two galaxies (NGC 5713 and NGC 7331) we have no information on extent. In these two cases we have listed [CI] luminosities ranging from that observed in a single beam to that appropriate to the implied maximum source diameter of $1 \mathrm{kpc}$.

The total [CI] luminosities of the observed galaxies cover a large range. Quiescent galaxies (NGC 7331, IC 342, Maffei 2, NGC 278, NGC 5713) have modest luminosities $\approx 1 \leq L_{[\mathrm{CI}]} \leq 5 \mathrm{Kkm} \mathrm{s}^{-1} \mathrm{kpc}^{2}$. Galaxies with a starburst nucleus (NGC 253, NGC 660, M 82, NGC 3628, NGC 6946) have luminosities $10 \leq L_{[\mathrm{CI}]} \leq$ $40 \mathrm{Kkm} \mathrm{s}^{-1} \mathrm{kpc}^{2}$. However, $\mathrm{M} 83$ has only $L_{[\mathrm{CI}]}=$ $3.6 \mathrm{~K} \mathrm{~km} \mathrm{~s}^{-1} \mathrm{kpc}^{2}$, although it is also a starburst galaxy. The highest luminosities $L_{[\mathrm{CI}]} \geq 50 \mathrm{~K} \mathrm{~km} \mathrm{~s}^{-1} \mathrm{kpc}^{2}$ are found in the active galaxies NGC 1068 and NGC 3079.

Interestingly, the ratio of the ${ }^{3} \mathrm{P}_{1}-{ }^{3} \mathrm{P}_{0}[\mathrm{CI}]$ and $J 2-1{ }^{13} \mathrm{CO}$ line strengths exhibits a similar behaviour. The [CI] line is stronger than $J=2-1{ }^{13} \mathrm{CO}$ in all galaxies except Maffei 2 and NGC 7331. The highest $[\mathrm{CI}] /{ }^{13} \mathrm{CO}$ ratios of about five belong to the active galaxies NGC 1068 and NGC 3079. Generally, the ${ }^{3} \mathrm{P}_{1}-{ }^{3} \mathrm{P}_{0}[\mathrm{CI}]$ line is weaker than the $J=4-3{ }^{12} \mathrm{CO}$ line, but not by much. In NGC 278 , NGC 3079, NGC 4826 and M 51, the two lines are roughly of equal strength. Only in Maffei 2 is the [CI] line much weaker.

\section{Comparison of $[\mathrm{Cl}]$ and ${ }^{13} \mathrm{CO}$ intensities}

In various studies of Galactic objects, the similarity of ${ }^{3} \mathrm{P}_{1}-{ }^{3} \mathrm{P}_{0}[\mathrm{CI}]$ and $J=2-1{ }^{13} \mathrm{CO}$ intensities and distribution is commented upon. Early such studies by the CSO group were reviewed by Keene et al. (1996). The CSO mapping of the Galactic molecular cloud complexes M 17 and Oph A show virtually identical line intensities for $[\mathrm{CI}]$ and ${ }^{13} \mathrm{CO}$ throughout. This is also found for most of the Orion Bar and OMC-1. The densest regions of Orion, however, show increasingly strong ${ }^{13} \mathrm{CO}$ emission whereas ${ }^{3} \mathrm{P}_{1}-{ }^{3} \mathrm{P}_{0}[\mathrm{CI}]$ intensities level off, yielding ever lower $[\mathrm{CI}] /{ }^{13} \mathrm{CO}$ intensity ratios down to about 0.4. A similar range of ratios $(0.3-1.1)$ was found by Jansen et al. (1996) towards the emission/reflection nebula IC 63. Keene et al. (1996) attributed such low values to the effects of enhanced UV radiation in photondominated regions (PDR's). This interpretation finds support in the results obtained by Plume et al. (1999) and Tatematsu et al. (1999) who used a reimaging device on the CSO to effectively obtain a larger beamsize suitable 

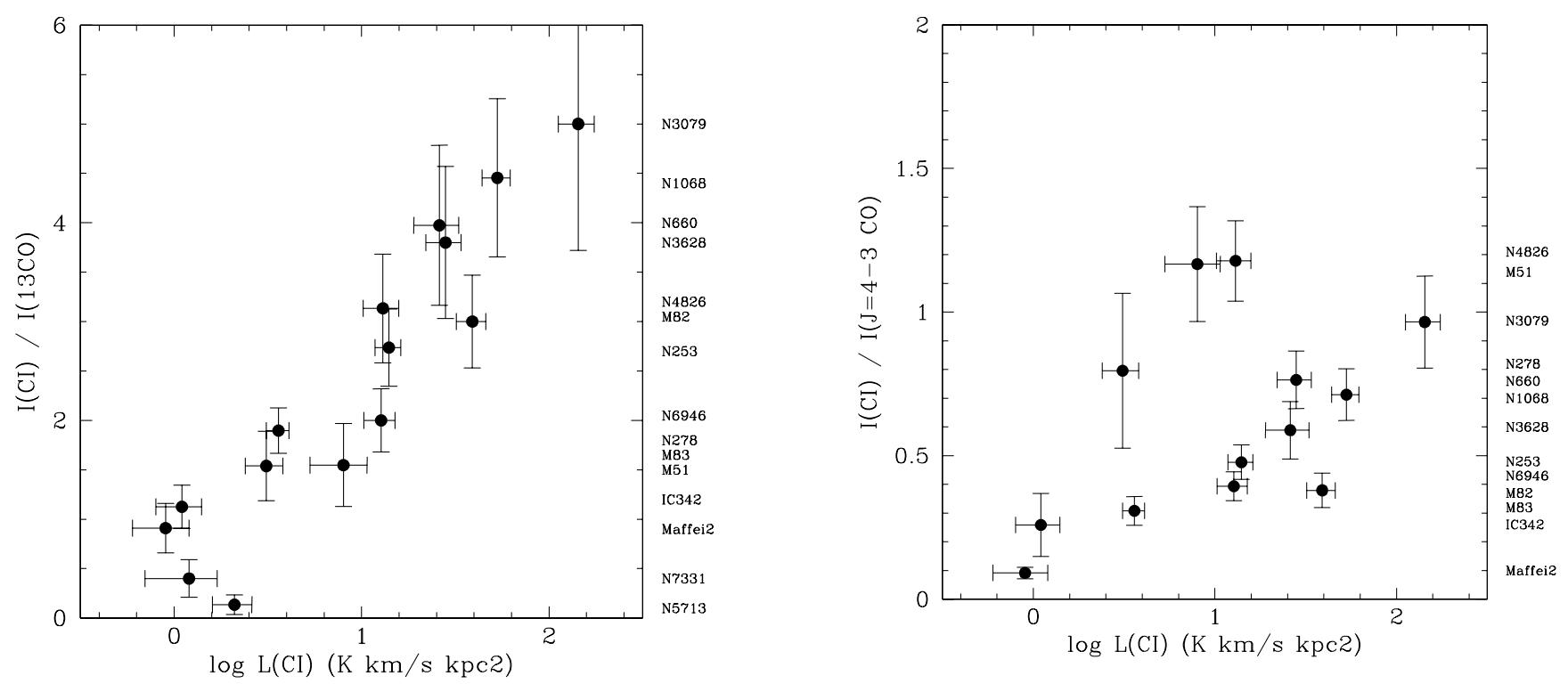

Fig. 2. Left: $[\mathrm{CI}] /\left(J=2-1^{13} \mathrm{CO}\right)$ ratios versus area-integrated $[\mathrm{CI}]$ luminosity $L[C I]$. Right: $[\mathrm{CI}] /\left(J=4-3^{12} \mathrm{CO}\right)$ ratios versus $L[C I]$. The $I([\mathrm{CI}]) / I\left({ }^{13} \mathrm{CO}\right)$ ratio appears to be a well-defined function of $\log L([\mathrm{CI}])$; the $I([\mathrm{CI}]) / I\left(4-3^{12} \mathrm{CO}\right)$ ratio is not. Galactic sources (not shown) would all be crowded together in the lower left corner.

for large-area mapping. Their maps of clouds associated with the low-UV sources TMC-1, L 134N and IC 5146 have fairly uniform ratios $\mathrm{I}[\mathrm{CI}] /{ }^{13} \mathrm{CO}=1.05 \pm 0.15$, as do the translucent regions of the dark cloud L 183 observed by Stark et al. (1996). In contrast, maps of the molecular clouds associated with the high-UV sources W3, NGC 2024, S140 and Cep A yield I[CI $] /{ }^{13} \mathrm{CO}$ ratios of about 0.5 for the bulk of the clouds. However, even here intensity ratios of about unity are found once again at cloud edges. The distribution of cloud-edge ratios even has a tail reaching a value of four. Only in a few globules associated with the Helix planetary nebula (Young et al. 1997) have such relatively high ratios of 3-5 also been found.

Our own data on star formation regions corroborate this: towards the Galactic HII regions W 58 and ON-1 (unpublished) as well as the LMC regions N 159 and N 160 (Bolatto et al. 2000) we find intensity ratios $[\mathrm{CI}] / J=$ $2-1=0.2-0.6$ for the PDR zones associated with these starforming regions. The two objects (W-58C and N 159South) where star formation has not yet progressed to a dominating stage, in contrast, yield ratios of about unity.

As Fig. 2 shows, only a few of the observed galaxy centers obey the same linear correlations between [CI] and ${ }^{13} \mathrm{CO}$ that characterize Galactic clouds. Fully two thirds of the galaxy sample has ${ }^{3} \mathrm{P}_{1}-{ }^{3} \mathrm{P}_{0}[\mathrm{CI}] / J=2-1{ }^{13} \mathrm{CO}$ ratios well in excess of unity; the galaxies thus have much stronger [CI] emission than the ${ }^{13} \mathrm{CO}$ intensity and the Galactic results would lead us to expect.

The galaxy sample, observed at $15^{\prime \prime}$ resolution, discussed by Gerin \& Phillips (2000) has only a little overlap with ours, but it shows the same effect: more than two thirds of the positions plotted in their Fig. 7 has a ratio $[\mathrm{CI}] /{ }^{13} \mathrm{CO} \geq 2$. For the galaxy NGC 891 , Gerin \& Phillips (2000) observed various positions along the major axis, in addition to the central region. At the distance of the galaxy, their beam corresponds to a linear size of $0.5 \mathrm{kpc}$. Whereas the [CI] intensity generally drops with increasing radius, the $[C I] /{ }^{13} \mathrm{CO}$ intensity ratio increases, or more specifically, this ratio increases from about 2 at the central positions brightest in [CI] to about $4-6$ at the disk positions weakest in [CI].

Qualitatively, low ratios are expected from regions which have low neutral carbon abundances. Low neutral carbon abundances will be found in high-UV environments where neutral carbon will become ionized, and in environments with high gas densities and column densities. Here, neutral carbon disappears because of the concomitant higher $\mathrm{CO}$ formation rates at high densities and the much more efficient $\mathrm{CO}$ (self)shielding at high column densities. Because of its lower abundance, ${ }^{13} \mathrm{CO}$ requires larger column densities for efficient shielding. Conversely, in environments characterized by low gas column densities and mild UV radiation fields, such as found in translucent clouds and at cloud edges, CO will be mostly dissociated, and most gas-phase carbon may be neutral atomic. The resultant relatively high neutral carbon abundance will then explain high $[\mathrm{CI}] /{ }^{13} \mathrm{CO}$ intensity ratios. In this framework, our observations and those obtained by Gerin \& Phillips (2000) imply that most of the emission from galaxy centers does not come from very dense, starforming molecular cloud cores.

\section{5. $[\mathrm{Cl}] / \mathrm{CO}$ modelling}

In a number of studies (e.g. Schilke et al. 1993; Tauber et al. 1995; Petitpas \& Wilson 1998) column densities have been calculated assuming [CI] and CO emission to occur under optically thin LTE conditions in the high-temperature limit. From Eqs. (1) and (2) by Tauber et al. (1995), it follows that:

$$
\left.I([\mathrm{CI}]) / I\left({ }^{13} \mathrm{CO}\right)=0.0063 A_{13}^{12} f\left(T_{\mathrm{ex}}\right) N[\mathrm{CI}]\right) / N(\mathrm{CO})
$$




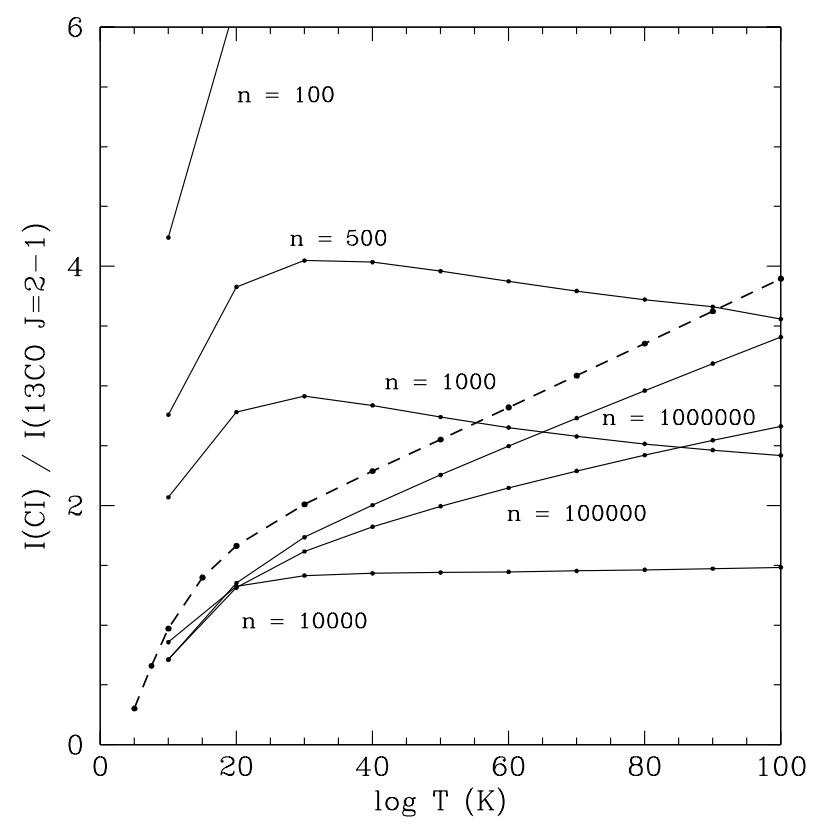

Fig. 3. Model line ratio $I(\mathrm{CI}) / I\left(J=2-1^{13} \mathrm{CO}\right)$ versus $T_{\text {kin }}$ resulting for equal column densities $N(\mathrm{CI}) / \mathrm{d} V=N(\mathrm{CO}) / \mathrm{d} V$ (solid lines). Curves for a range of total gas densities are marked in particles per cc. Dashed line: the ratio $I(\mathrm{CI}) / I(J=$ $\left.2-1{ }^{13} \mathrm{CO}\right)$ versus $T_{\text {ex }}$, likewise requiring equal column densities $N(\mathrm{CI}) / \mathrm{d} V=N(\mathrm{CO}) / \mathrm{d} V$ but assuming optically thin LTE conditions.

where

$f\left(T_{\text {ex }}\right)=T_{\text {ex }} /\left(\mathrm{e}^{7 / T_{\text {ex }}}+3 \mathrm{e}^{-16.6 / T_{\text {ex }}}+5 \mathrm{e}^{-55.5 / T_{\text {ex }}}\right)$

while we assume an isotopic abundance $A_{13}^{12}=$ $\left[{ }^{12} \mathrm{CO}\right] /\left[{ }^{13} \mathrm{CO}\right]=40$ (cf. Mauersberger \& Henkel 1993; Henkel et al. 1998). In Fig. 3 we have marked by a dashed line the expected line intensity ratios for the case $N[\mathrm{CI}]) / N(\mathrm{CO})=1$. In order to verify the correctness of the assumptions of low optical depth and LTE, we have used the Leiden radiative transfer models described by Jansen (1995) and Jansen et al. (1994) to calculate for gas volume densities ranging from $10^{2} \mathrm{~cm}^{-3}$ to $10^{6} \mathrm{~cm}^{-3}$ the $[\mathrm{CI}] /{ }^{13} \mathrm{CO}$ line intensity ratio corresponding to unit column density ratios. The calculation was performed for a representative value of the column density, $N[\mathrm{CI}] / \mathrm{d} V=N(\mathrm{CO}) / \mathrm{d} V=1 \times 10^{17} \mathrm{~cm}^{-2} \mathrm{~km} \mathrm{~s}^{-1}$ (cf. Israel \& Baas 2001). Note that in this calculation, the temperature parameter is the kinetic temperature $T_{\text {kin }}$ instead of the excitation temperature $T_{\mathrm{ex}}$. For neutral carbon, the two are not very different under the conditions considered, but for ${ }^{13} \mathrm{CO}$ the excitation temperature is generally much lower than the kinetic temperature over most of the relevant range. Under LTE conditions, the $[\mathrm{CI}] /{ }^{13} \mathrm{CO}$ ratio continuously increases with temperature $T_{\text {ex }}$. In contrast, the radiative transfer calculation shows that this ratio is only weakly dependent on temperature above $T_{\text {kin }} \approx 30 \mathrm{~K}$, and in fact decreases slowly with increasing temperature for densities up to $n\left(\mathrm{H}_{2}\right) \approx 10^{4}$. As Fig. 3 illustrates, the assumption of comparable excitation temperatures for ${ }^{13} \mathrm{CO}$ and [CI] is valid only for very high densities $n\left(\mathrm{H}_{2}\right)>10^{6} \mathrm{~cm}^{-3}$ which are unlikely to apply to our observed sample.

\section{6. $[\mathrm{Cl}]$ and $\mathrm{CO}$ column densities}

To further investigate the physical conditions characterizing the central gas clouds that give rise to the observed emission, we have plotted for our galaxy sample the $[\mathrm{CI}] / J=2-1^{13} \mathrm{CO}$ line intensity ratio versus the $[\mathrm{CI}] / J=$ $4-3^{12} \mathrm{CO}$ line ratio. For comparison purposes, we have added points corresponding to a few Galactic starforming regions (White \& Sandell 1995; Israel \& Baas, unpublished), the N 159/N 160 starforming complex in the Large Magellanic Cloud (Bolatto et al. 2000), and the Milky Way Center (Fixsen et al. 1999). As the latter do not list ${ }^{13} \mathrm{CO}$ intensities, we have assumed a $J=2-1{ }^{12} \mathrm{CO} /{ }^{13} \mathrm{CO}$ intensity ratio of 8.5 , which is the mean value we find for the galaxies observed by us (Israel \& Baas 1999, 2001, as well as papers in preparation).

To put the observed points in context, we have used the Leiden radiative transfer models to calculate the same line intensity ratio in a grid with gas densities in the range $n=500-10000 \mathrm{~cm}^{-3}$, kinetic temperatures in the range $T_{\text {kin }}=10-150 \mathrm{~K}$ and $\mathrm{CO}$ column densities $N(\mathrm{CO}) / \mathrm{d} V=$ $0.3,1.0$ and $3.0 \times 10^{17} \mathrm{~cm}^{-2} / \mathrm{km} \mathrm{s}^{-1}$ respectively. We considered $N([\mathrm{CI}]) / N(\mathrm{CO})$ abundance ratios of $0.1,0.3,1.0$ and 3.0 respectively. The results are shown in Fig. 4, always assuming an isotopic ratio $\left[{ }^{12} \mathrm{CO}\right] /\left[{ }^{13} \mathrm{CO}\right]=40$. Small variations in the assumed isotopic ratio lead to small shifts in the various curves depicted in Fig. 4, mostly along lines of constant temperature.

It is immediately clear from Fig. 4 that the predicted $[\mathrm{CI}] /{ }^{13} \mathrm{CO}$ intensity ratio is roughly proportional to the $N([\mathrm{CI}]) / N(\mathrm{CO})$ abundance ratio at any given gas-density. Variation of the actual CO column density by over an order of magnitude or variation of the gas kinetic temperature has very little effect on the line intensity ratio except at the highest densities and column densities where saturation effects caused by high optical depths become dominant. At given column densities, however, the $[\mathrm{CI}] /{ }^{13} \mathrm{CO}$ intensity ratio does depend on the gas-density and is roughly inversely proportional to $\sqrt{n}$. The $[\mathrm{CI}] / J=$ $4-3{ }^{12} \mathrm{CO}$ intensity ratio strongly varies as a function of gas kinetic temperature and density, as well as column density.

Further inspection of Fig. 4 shows that the starforming regions in the Milky Way and the LMC are found distributed along curves that mark neutral carbon versus $\mathrm{CO}$ abundances $\left.N\left(\mathrm{C}^{\circ}\right) / N C O\right) \approx 0.1-0.3$. The galaxy center ratios, in contrast, mostly seem to imply significantly higher neutral carbon abundances. Only the point representing the quiescent bulge of NGC 7331 appears to be associated with an equally low carbon abundance. Depending on the assumed value of the total gas density, centers of quiescent galaxies are associated with carbon abundances $\left.N\left(\mathrm{C}^{\circ}\right) / N \mathrm{CO}\right) \approx 0.3\left(n=500 \mathrm{~cm}^{-3}\right)$ to $1.0\left(n=10^{4} \mathrm{~cm}^{-3}\right)$. This is consistent with earlier determinations such as $\left.N\left(\mathrm{C}^{\circ}\right) / N \mathrm{CO}\right) \approx 0.8(-0.4,+0.7)$ for 

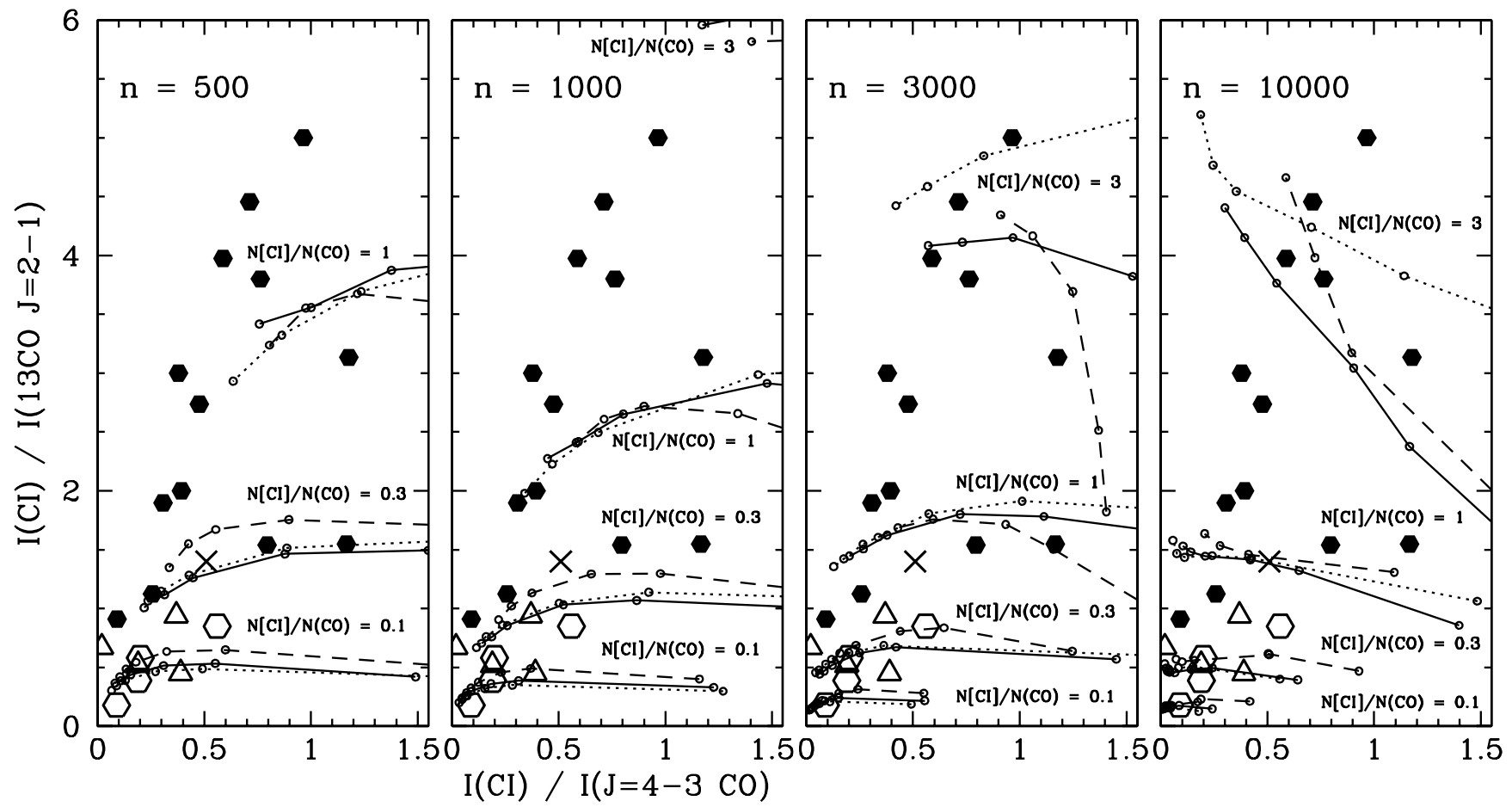

Fig. 4. Observed line intensity ratios $[\mathrm{CI}] /{ }^{13} \mathrm{CO}$ versus $[\mathrm{CI}] / \mathrm{CO} 4-3$ compared to radiative-transfer model ratios at selected gas densities, for a range of temperatures and column densities. All model calculations assume an isotopic ratio $\left[{ }^{12} \mathrm{CO}\right] /\left[{ }^{13} \mathrm{CO}\right]=$ 40. Galaxy centers are marked by filled hexagons, LMC star formation regions N 159 and N 160 by open hexagons, Galactic star formation regions $\mathrm{W} 58$ and $\mathrm{ON}-1$ by open triangles and the Milky Way Center by a cross. Lines indicate families of column density ratios $N[\mathrm{CI}] / N(\mathrm{CO})=0.1,0.3,1$ and 3 . Within each family, dotted line corresponds to $N(\mathrm{CO}) / \mathrm{d} V=3 \times$ $10^{16} \mathrm{~cm}^{-2} / \mathrm{km} \mathrm{s}^{-1}$, solid line to $N(\mathrm{CO}) / \mathrm{d} V=1 \times 10^{17} \mathrm{~cm}^{-2} / \mathrm{km} \mathrm{s}^{-1}$ and dashed line to $N(\mathrm{CO}) / \mathrm{d} V=3 \times 10^{17} \mathrm{~cm}^{-2} / \mathrm{km} \mathrm{s}^{-1}$. Temperatures of (from left to right) 150, 100, 60, 30, 20 and $10 \mathrm{~K}$ are marked by small open circles on each curve.

the Milky Way (Serabyn et al. 1994). In contrast, active galaxies have $\mathrm{C}^{\circ}$ column densities well exceeding $\mathrm{CO}$ column densities independent of the gas parameters assumed. The diagonal distribution of galaxy points roughly follows lines of constant kinetic temperature. The corresponding temperature value varies as a function of density $n$ and column density $(N): T_{\text {kin }}>150 \mathrm{~K}$ for $n=500 \mathrm{~cm}^{-3}$, whereas $T_{\text {kin }}=30-60 \mathrm{~K}$ for $n=0.3-1.0 \times 10^{4} \mathrm{~cm}^{-3}$, $N<10^{17} \mathrm{~cm}^{-2} / \mathrm{km} \mathrm{s}^{-1}$. Only the high-density models imply a kinetic temperature range covering the fairly narrow dust temperature range $33 \mathrm{~K} \leq T_{\mathrm{d}} \leq 52 \mathrm{~K}$ characterizing these galaxy centers (Smith \& Harvey 1996). This can be taken as a suggestion that at least the molecular carbon monoxide emission from galaxy centers arises mostly from warm, dense gas as opposed to either hot, tenuous gas or cold, very dense gas. Possible exceptions to this are NGC 278 and in particular NGC 7331, M 51 and NGC 4826 which occupy positions in the diagrams of Fig. 4 suggesting low temperatures $T_{\text {kin }}=10-20 \mathrm{~K}$ and consistent with the full density range including the highest densities.

For M 82, Stutzki et al. (1997) estimated from the directly observed ${ }^{3} \mathrm{P}_{2}-{ }^{3} \mathrm{P}_{1}[\mathrm{CI}] /{ }^{3} \mathrm{P}_{1}-{ }^{3} \mathrm{P}_{0}[\mathrm{CI}]$ line ratio a density $n \geq 10^{4} \mathrm{~cm}^{-3}$ and a temperature $T=50$ $100 \mathrm{~K}$. This is in very good agreement with our estimates. However, the $I([\mathrm{CI}]) / I\left({ }^{13} \mathrm{CO}\right)$ ratio of three suggests an abundance $N[\mathrm{CI}] / N(\mathrm{CO})=2$, i.e. four times higher than estimated by Stutzki et al. (1997), although not ruled out by their results - see also Schilke et al. (1993).

\section{7. [CI], [CII] and FIR intensities}

Parameters indicating a warm and dense gas is more or less what is expected if the emission arises from photondominated region (PDR's - see e.g. Kaufman et al. 2000). In order to explore this possibility, we have produced Fig. 5, which presents a comparison of the ${ }^{3} \mathrm{P}_{1}-{ }^{3} \mathrm{P}_{0}[\mathrm{CI}]$ and ${ }^{2} \mathrm{P}_{3 / 2}{ }^{2} \mathrm{P}_{1 / 2}[\mathrm{CII}]$ line and far-infrared continuum intensities; this Fig. 5 is directly comparable to Fig. 8 by Gerin \& Phillips (2000) who performed a similar comparison. The majority of the [CII] intensities were taken from Stacey et al. (1991), as were the [CII]/FIR ratios. The Milky Way data were taken from Fixsen et al. (1999), those for the LMC from Israel et al. (1996) and Bolatto et al. (2000), and those for NGC 278 from Kaufman et al. (2000), who use this galaxy as an example for the application of their PDR model calculations. The [CII] measurements by Stacey et al. (1991) were obtained in a $55^{\prime \prime}$ aperture, i.e. much larger than the $[\mathrm{CI}]$ beam. We have therefore used the area-integrated [CI] intensity, and assumed that all [CII] emission from the central source was contained within the $55^{\prime \prime}$ aperture. As the extent of the central $[\mathrm{CI}]$ source is always less than this, that seems to 


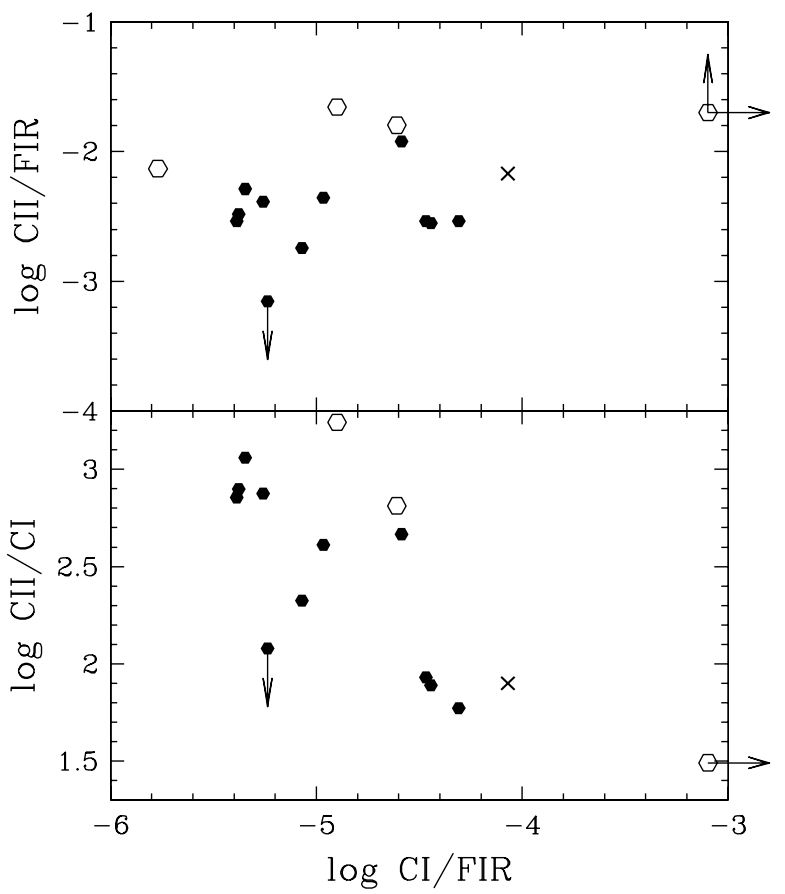

Fig. 5. Ionized carbon $[\mathrm{CII}]$ line to far-infrared continuum (FIR) ratio (top) and $[\mathrm{CII}] /[\mathrm{CI}]$ line ratio (bottom) as a function of neutral carbon [CI] line to FIR ratio. In both diagrams, the position of the Milky Way center is marked by a cross and the positions of Magellanic Cloud objects by open hexagons.

be a reasonable assumption. If the [CII] emission is more extended than that of $[\mathrm{CI}]$, the relevant $[\mathrm{CII}] /[\mathrm{CI}]$ ratio in Fig. 5 should be lowered correspondingly. However, we do not believe such a correction will change the picture significantly. The $[\mathrm{CI}] / \mathrm{FIR}$ ratio was obtained from the $[\mathrm{CII}] /[\mathrm{CI}]$ and $[\mathrm{CII}] / \mathrm{FIR}$ ratio; thus $[\mathrm{CI}] / \mathrm{FIR}$ may be somewhat higher than plotted.

In Fig. 5, there is no longer a clear distinction between various types of objects such as we found in Fig. 4. Rather, the [CII], [CI] and FIR intensities define a distribution in which LMC star formation regions, low-activity galaxy centers and high-activity galaxy centers are all intermingled. Nevertheless, the result shown in Fig. 5 bears a close resemblance to the the results obtained by Gerin \& Phillips (2000). As the [CI]/FIR ratio increases, so does $[\mathrm{CII}] / \mathrm{FIR}$, but not the $[\mathrm{CII}] /[\mathrm{CI}]$ ratio which decreases with increasing [CI]/FIR. Qualitatively, this may be explained by PDR process along the line discussed by Gerin \& Phillips (2000). The horizontal location of the points in the two diagrams suggest fairly intense PDR radiation fields of about 300 to 1000 times the average UV radiation field in the Solar Neighbourhood. For the merger galaxy NGC 660 we have only upper limits (log [CII]/FIR < $-3.2, \log [\mathrm{CII}] /[\mathrm{CI}]<2.1)$ which place this galaxy in the same diagram positions as the ultraluminous mergers Arp 220 and Mrk 231 observed by Gerin \& Phillips, which correspond to strong radiation fields and very high gas densities.

The PDR models shown in Fig. 8 by Gerin \& Phillips provide the highest $[\mathrm{CII}] / \mathrm{FIR}$ ratios for model gas densities $n=10^{3}-10^{4} \mathrm{~cm}^{-3}$. Fully half of our observed ratios are well above the corresponding curves, although they are not quite as high as the ratios observed for the three LMC starforming regions. Note that (the limits to) the quiescent cloud LMC N159-S in Fig. 5 likewise suggest high densities but only weak radiation fields, in good agreement with Israel et al. (1996) and Bolatto et al. (2000). For many of the galaxies and for the LMC starforming regions, the ratio of $[\mathrm{CII}]$ to $[\mathrm{CI}]$ intensities appears to be higher than predicted by the PDR models considered. For the LMC objects, this was already noted and discussed by Israel et al. (1996). They explain this situation by an increased mean free pathlength of energetic UV photons due the lower metallicity of the LMC. However, galaxy centers have, if anything, a higher metallicity (see Zaritsky et al. 1994). A possible explanation for the apparently similar behaviour of many galaxy centers may be a greater degree of filamentary or cirruslike structure. In spite of high metallicities, this would still allow for an effectively increased penetration depth of UV photons. If enhanced exposure results in a significantly larger fraction of carbon atoms becoming ionized, it would explain higher [CII] to [CI] emission ratios.

So far we have assumed homogeneous media, i.e. we have assumed all CO, [CI], [CII] and FIR emission to originate from the same volume. This provides in a relatively simple manner good estimates of the physical parameters characterizing the interstellar medium in the observed galaxy centers.

The LMC observations, which correspond to linear resolutions one to two orders of magnitude higher than the galaxy center observations, illustrate that homogeneity is not the case. The maps shown by Israel et al. (1996) and Bolatto et al. (2000) show that different locations in the observed regions are characterized by strongly different emission ratios indicating domination by different ISM phases (i.e. neutral atomic, ionized, molecular). A similar state of affairs applies to the Galactic Center region (Dahmen et al. 1998). Ideally, the observations should thus be modelled by physical parameters varying as a function of location in a complex geometry. Practically, we may approach reality by assuming the presence of a limited number of distinct gas components. The analysis of multitransition ${ }^{12} \mathrm{CO},{ }^{13} \mathrm{CO}$ and $[\mathrm{CI}$ ] observations of galaxy centers such as those of NGC 7331, M 83 and NGC 6946 (Israel \& Baas 1999, 2001) suggests that, within the observational errors, good fits to the data can be obtained by modelling with only two components: one being dense and relatively cool, the other being relatively tenuous and warm.

The galaxy points in Fig. 5 can all be reproduced by assuming appropriate combinations of dense and cold gas (having high [CI]/FIR and [CII]/FIR ratios) with strongly irradiated gas of lower density (low $[\mathrm{CI}] / \mathrm{FIR}$ and high $[\mathrm{CII}] /[\mathrm{CI}]$ ratios). The distribution of points in Fig. 5 would thus not directly indicate the physical condition of the radiating gas, but rather the relative filling factors of the two components. A similar argument can be made to solve the apparent discrepancy between the relatively 
high kinetic temperatures suggested by Fig. 4 and the more modest dust temperatures referred to before. In the same vein, a multi-component solution requires somewhat lower beam-averaged [CI]/CO abundances than suggested by Fig. 4. The dataset presented in this paper is, however, not sufficiently detailed to warrant a more quantitative analysis such as we have presented for NGC 7331, M 83 and NGC 6946 (Israel \& Baas 1999, 2001), and will present for half a dozen more in forthcoming papers.

\section{Conclusions}

1. We have measured the emission from the $492 \mathrm{GHz}$ line corresponding to the ${ }^{3} \mathrm{P}_{1}-{ }^{3} \mathrm{P}_{0}[\mathrm{CI}]$ transition in the centers of fifteen nearby spiral galaxies. In the same galaxies, we have also measured $J=4-3{ }^{12} \mathrm{CO}$ and $J=2-1^{13} \mathrm{CO}$ intensities for comparison with the ${ }^{3} \mathrm{P}_{1-}$ ${ }^{3} \mathrm{P}_{0}[\mathrm{CI}]$ line within the framework of radiative transfer models.

2. Rather unlike Galactic sources, the external galaxy centers have $[\mathrm{CI}]$ intensities generally exceeding $J=$ $2-1{ }^{13} \mathrm{CO}$ intensities, and in a number of cases approaching $J=4-3{ }^{12} \mathrm{CO}$ intensities.

3. The highest area-integrated (i.e. total central) $[\mathrm{CI}]$ luminosities are found in the active galaxies NGC 1068 and NGC 3079. Slightly lower luminosities occur in strong starburst nuclei, such as those of NGC 3628 and NGC 6946. Quiescent and weak-starburst nuclei have [CI] luminosities an order of magnitude lower.

4. The observed [CI], ${ }^{13} \mathrm{CO}$ and ${ }^{12} \mathrm{CO}$ line ratios, interpreted within the context of radiative transfer models, suggest that the bulk of the observed emission arises in gas with densities $n \geq 3000 \mathrm{~cm}^{-3}$ and kinetic temperatures $T_{\text {kin }} \approx 30-60 \mathrm{~K}$. Depending on the actual density $n$, most galaxy centers should have abundances $N([\mathrm{CI}]) / N(\mathrm{CO})=1-3$, i.e. $[\mathrm{CI}]$ columns just exceeding those of CO. Only relatively quiescent galaxy centers such as those of Maffei 2, IC 342 and NGC 7331 have abundances $N([\mathrm{CI}]) / N(\mathrm{CO}) \approx 0.3-1.0$ and are dominated by $\mathrm{CO}$ just as the comparison starforming regions in the Milky Way and the LMC.

5. The observed $[\mathrm{CI}]$ intensities, together with literature [CII] line and far-infrared continuum data, likewise suggest that a significant fraction of the emission originates in medium-density gas $\left(n=10^{3}-10^{4} \mathrm{~cm}^{-3}\right)$, subjected to radiation fields of various strengths ranging from a few times to several thousand times the local Galactic radiation field.

Acknowledgements. We are indebted to Ewine van Dishoeck and David Jansen for providing us with their their statistical equilibrium calculation models. We also thank Maryvonne Gerin and Tom Phillips for communicating to us their neutral carbon measurements of galaxies well before publication.

\section{References}

Bolatto, A. D., Jackson, J. M., Israel, F. P., Zhang, X., \& Kim, S. 2000, ApJ, 545, 234
Büttgenbach, T. H., Keene, J., Phillips, T. G., \& Walker, C. K. 1992, ApJ, 397, L15

Dahmen, G., Hüttemeister, S., Wilson, T. L., \& Mauersberger, R. 1998, A\&A, 331, 959

Fixsen, D. J., Bennet, C. L., \& Mather, J. C. 1999, ApJ, 526, 207

Garnett, D. R., Skillman, E. D., Dufour, R. J., et al. 1995, ApJ, 443, 64

Gerin, M., \& Phillips, T. G. 2000, ApJ, 537, 644

Harrison, A., Puxley, P., Russell, A., \& Brand, P. 1995, MNRAS, 277, 413

Henkel, C., Chin, Y.-N., Mauersberger, R., \& Whiteoak, J. B. 1998, A\&A, 329, 443

Hollenbach, D. J., \& Tielens, A. G. G. M. 1999, Rev. Mod. Phys., 71, 173

Israel, F. P. 1997, A\&A, 328, 471

Israel, F. P., White, G. J., \& Baas, F. 1995, A\&A, 302, 343

Israel, F. P., Maloney, P. R., Geis, N., et al. 1996, ApJ, 465, 738

Israel, F. P., Tilanus, R. P., \& Baas, F. 1998, A\&A, 339, 398

Israel, F. P., \& Baas, F. 1999, A\&A, 351, 10

Israel, F. P., \& Baas, F. 2001, A\&A, 371, 433

Jansen, D. J. 1995, Ph.D. Thesis, University of Leiden (NL)

Jansen, D. J., van Dishoeck, E. F., \& Black, J. H. 1994, A\&A, 282,605

Jansen, D. J., van Dishoeck, E. F., Keene, J., Boreiko, R. T., \& Betz, A. L. 1996, A\&A, 309, 899

Kaufman, M. J., Wolfire, M. G., Hollenbach, D. J., \& Luhman, M. L. 1999, ApJ, 527, 795

Keene, J., Blake, G. A., Phillips, T. G., Huggins, P. J., \& Beichman, C. A. 1985, ApJ, 299, 967

Keene, J., Lis, D. C., Phillips, T. G., \& Schilke, P. 1996, in Molecules in Astrophysics, ed. E. F., van Dishoeck, IAU Symp., 178, 129

Mauersberger, R., \& Henkel, C. 1993, Rev. Mod. Astron., 6, 69

Petitpas, G. R., \& Wilson, C. D. 1998, ApJ, 503, 219

Plume, R., Jaffe, D. T., Tatematsu, K., Evans, N. J., \& Keene, J. 1999, ApJ, 512, 768

Serabyn, E., Keene, J., Lis, D. C., \& Phillips, T. G. 1994, ApJ, 424, L95

Smith, B. J., \& Harvey, P. M. 1996, ApJ, 468, 193

Sodroski, T. J., Odegard, N., \& Dwek, E., et al. 1995, ApJ, 452,262

Schilke, P., Carlstrom, J. E., Keene, J., \& Phillips, T. G. 1993, ApJ, 417, L67

Stacey, G. J., Geis, N., Genzel, R., et al. 1991, ApJ, 373, 423

Stark, R., Wesselius, P. R., van Dishoeck, E. F., \& Laureijs, R. J. 1996, A\&A, 311, 282

Stutzki, J., Graf, U. U., Haas, S., et al. 1997, ApJ, 477, L33

Tatematsu, K., Jaffe, D. T., Plume, R., Evans, N. J., \& Keene, J. 1999, ApJ, 526, 295

Tauber, J. A., Lis, D. C., Keene, J., Schilke, P., \& Büttgenbach, T. H. 1995, A\&A, 297, 567

van Dishoeck, E. F. 1998, in The Molecular Astrophysics of Stars and Galaxies - A Volume Honouring Alex Dalgarno, ed. T. W. Hartquist, \& D. A. Williams (Oxford University Press), in press

van Dishoeck, E. F., \& Black, J. H. 1988, ApJ, 334, 771

White, G. J., Ellison, B., Claude, S., Dent, W. R. F., \& Matheson, D. N. 1994, A\&A, 284, L23

White, G. J., \& Sandell, G. 1995, A\&A, 299, 179

Young, K., Cox, P., Huggins, P. J., Forveille, T., \& Bachiller, R. 1997, ApJ, 482, 101

Zaritsky, D., Kennicutt, R. C., \& Huchra, J. P. 1994, ApJ, 420, 87 